\title{
ASOCIACIÓN ENTRE LA EDAD Y TIPO DE LESIÓN ENCONTRADA POR ENDOSCOPIA BAJA EN PACIENTES CON HEMORRAGIA DIGESTIVA BAJA, HOSPITAL SANTA ROSA-2015
}

\author{
ASSOCIATION BETWEEN AGE AND TYPE OF INJURY FOUND BY LOW ENDOSCOPY IN PATIENTS WITH LOW \\ DIGESTIVE HEMORRHAGE, SANTA ROSA HOSPITAL-2015
}

María Elena Sandoval-Pereda ${ }^{1, a}$, Jorge Burgos-Miranda ${ }^{2, b, c}$

\begin{abstract}
RESUMEN
Objetivo: Determinar la asociación entre la edad con los tipos de lesiones encontradas en pacientes con diagnóstico de hemorragia digestiva baja sometidas a endoscopia baja en el servicio de Gastroenterología del hospital Santa Rosa. Métodos: Estudio observacional, analítico, retrospectivo, se recopiló información de los informes de endoscopia digestiva baja y los archivos de historia clínica del servicio de Gastroenterología. Las variables: edad, sexo, tipo de lesión y localización anatómica de la lesión; se resumieron a fichas para su uso estadístico utilizando los programas de SSPS versión 22 y Excel para los gráficos y tablas, se usó la prueba de Chi cuadrado, con un valor de $p<0,05$ como estadísticamente significativo. Resultados: Muestra significativa de 180 pacientes con edad promedio de 62 años, dividida en G1: (18-34 años) con 10\% de la muestra, predomina el examen endoscópico sin evidencia de lesión, seguido de hemorroides, G2: (35-64 años) con 38\% de la muestra, predomina hemorroides externas, G3: (65 a más) con 52\% de la muestra, predomina hemorroides internas, con respecto al sexo $60 \%$ son de sexo femenino; la localización anatómica más frecuente se encontró a nivel del recto-ano. Además la asociación entre pacientes <35 años y 35 años a más respecto a la presencia o no de lesión endoscópica, nos generó un OR de 18.514 el cual fue estadísticamente significativo. Conclusión: Se concluye que existe asociación entre la edad y/o el grupo etario con el tipo de lesión endoscópica encontrada, predominando el grupo etario GIII que corresponde a los adultos mayores de 65 años, en el que se encontró la mayor cantidad de hemorroides internas y externas; habiendo asociación entre la edad del paciente con la presencia de lesión endoscópica, con un OR de 18.5; lo cual nos indica que existe 18.5 veces más riesgo que un paciente a mayor edad presente hemorragia digestiva baja encontrada por endoscopia baja, por lo cual esta relación fue estadísticamente significativa.
\end{abstract}

Palabras clave: Hemorragia digestiva baja; Localización anatómica de la lesión; Tipo de lesión endoscópica. (fuente: DeCS BIREME)

\begin{abstract}
Objective: To determine the association between age with the types of lesions found in patients diagnosed with low digestive bleeding undergoing low endoscopy in the Gastroenterology service of Santa Rosa Hospital. Methods: Observational, analytical, retrospective study, information was collected from the reports of low digestive endoscopy and medical history files of the Gastroenterology service. Variables: age, sex, type of injury and anatomical location of the lesion; it was summarized to cards for their statistical use using the SSPS version 22 and Excel programs for the graphs and tables, Chi square test was used, with a value of $p<0.05$ as statistically significant. Results: Sample of 180 patients with an average age of 62 years, divided into G1: (18-34 years) with 10\% of the sample, the endoscopic examination predominates without evidence of injury, followed by hemorrhoids, G2: (35-64 years) with 38\% of the sample, external hemorrhoids predominate, G3: (65 to more) with $52 \%$ of the sample, internal hemorrhoids predominate, with respect to sex $60 \%$ are female; The most frequent anatomic location was found at the level of the rectum-anus. In addition, the association between patients $<35$ years and 35 years old more than the presence or absence of endoscopic lesion, generated an OR 18,514 which was statistically significant. Conclusion: It is concluded that there is an association between age and / or age group with the type of endoscopic lesion found, predominantly the age group GIII corresponding to adults over 65 years of age, in which the highest number of internal hemorrhoids was found. and external; there being an association between the age of the patient with the presence of endoscopic lesion, with an OR of 18.5; this indicates that there is an 18.5 times higher risk than an older patient with lower gastrointestinal bleeding found by low endoscopy, which is why this relationship was statistically significant.
\end{abstract}

Key words: Low digestive hemorrhage; Anatomical location of the lesion; Type of endoscopic injury. (source: MeSH NLM)

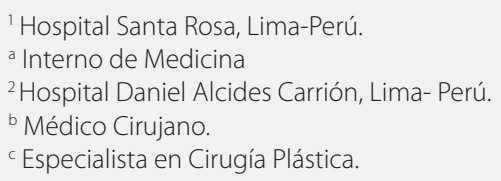

Citar como: María Elena Sandoval-Pereda, Jorge Burgos-Miranda. Asociación entre la edad y tipo de lesión encontrada por endoscopia baja en pacientes con hemorragia digestiva baja, Hospital Santa Rosa-2015.[Artículo Original].2019;19(1):32-39. (Enero 2019). DOl 10.25176/RFMH.v19.n1.1790 


\section{INTRODUCCIÓN}

En el Perú el $10-15 \%$ de todos los sangrados digestivos que presenta la población general corresponden a hemorragia digestiva baja(HDB); sin embargolas cifras son mayores (20-30\%) en el adulto mayor, también la tasa de hospitalización por HDB se incrementa dramáticamente de 200 veces más después de los 80 años'.

La mayor incidencia de HDB en adultos mayores está relacionada al incremento de la prevalencia de enfermedad diverticular de colon y angiodisplasia con la edad. La búsqueda del origen de la HDB en algunas ocasiones es difícil por el extenso territorio que conforman el intestino delgado y grueso ${ }^{2}$.

Varios estudios han concluido que la edad influye en la presencia o no de hemorragia digestiva baja, ya que a mayor edad es más frecuente encontrar casos de hemorragia digestiva baja a nivel mundial ${ }^{3-6}$.

Es de considerar que la hemorragia digestiva baja de tipo aguda se autolimita en el $85 \%$ de los casos, en el $15 \%$ restante permanece activo o resurgido, estos últimos casos requieren una gestión verdaderamente rápida en vista del hecho de que alrededor del $50 \%$ requieren tratamiento quirúrgico de emergencia ${ }^{7}$.

En la actualidad, la incidencia anual de pacientes que presentan HDB grave es de 20,5 a 27 casos/ 100.000 habitantes y su mortalidad global es inferior al $5 \%$ del total. Su predominio es ligeramente mayor en el sexo masculino y por lo general se presenta en edades avanzadas de la vida, por lo general a los 75 años a más, y suele asociarse con frecuencia a enfermedades graves o incluso con antecedentes de intervenciones quirúrgicas ${ }^{8-10}$.

La hemorragia digestiva baja representa de por si un serio problema de salud para la comunidad actual, no solo representan un serio problema en el ámbito de la morbimortalidad, sino también un problema presupuestal para un sistema de salud que lucha por sostenerse año tras año, debido a la gran demanda de servicios de salud por dicha causa ${ }^{11}$.

Dentro del universo de las hemorragias digestivas bajas existen numerosas etiologías, si bien la mayoría son auto limitadas o problemas leves propios de la edad como inflamación y diverticulosis, también existen etiologías de pronóstico reservado como el cáncer de colon por ejemplo, lo cual representa un gran sufrimiento y dolencia tanto física como psicológica para el paciente en cuestión, así como un extenso gasto en fármacos y procedimientos financiados en su mayoría por el estado ${ }^{12}$.

En el presente trabajo, se busca conocer mejor la HDB, así como los tipos de lesiones asociadas a diversas patologías del cual la HDB representa únicamente un signo clínico importante, todo esto desde un punto de vista estadístico y basado en evidencias encontradas en colonoscopias avaladas por especialistas en el área y veremos a que etiología / enfermedad nos lleva, para así poder tener una sospecha clínica asociada a edad, sexo, con un base científica de evidencias de todo un año, el cual hemos escogido será el 2015 debido a su proximidad.

Por lo mencionado, en la presente investigación buscamos conocer la asociación entre la edad y el tipo de lesión encontrada en pacientes con diagnóstico de hemorragia digestiva baja sometidos a endoscopia baja en el servicio de Gastroenterología del hospital Santa Rosa durante el año 2015

\section{MÉTODOS}

Se realizó un estudio de tipo observacional, analítico, y retrospectivo, donde se recopiló información proveniente de los informes de endoscopia digestiva baja y los archivos de historia clínica del servicio de Gastroenterología del hospital Santa Rosa. Se tuvo en cuenta las siguientes variables: edad, sexo, tipo de lesión y localización anatómica de la lesión; lo cual fue resumido a fichas para su uso estadístico utilizando los programas de SSPS versión 22, Excel, con lo que se determinó la asociación en los gráficos, tablas, usando la prueba de Chi cuadrado, con un valor de $p<0,05$ como estadísticamente significativo para obtener los resultados necesarios para la investigación.

La ejecución de este trabajo comenzó con la exposición y correcciones realizadas del proyecto del mismo a las autoridades del Instituto de Investigación en Ciencias Biomédicas (INICIB) de la Facultad de Medicina Humana de la Universidad Ricardo Palma, hasta esperar su aprobación. Luego solicitamos al Director del hospital Santa Rosa el permiso y las facilidades para acceder a las historias clínicas e informes de endoscopia baja, desde enero a diciembre del 2015, y una vez aceptado el permiso, se procedió a las coordinaciones con el personal de archivo y se realizó la recolección de datos a través de la recopilación de información procedente de las historias clínicas e informes de endoscopia baja del servicio de Medicina General del hospital Santa Rosa durante el año 2015; los cuales fueron transcritos a una ficha de recolección de datos para su uso.

Luego de obtener los datos por medio de las historias clínicas e informes de endoscopia baja, se tabularon y se presentaron en gráficos y tablas seleccionados para tal fin. Utilizamos la prueba estadística Chi Cuadrado para efectuar el análisis de la muestra, con un $p<0,05$ como significativo y un intervalo de confianza del $95 \%$. 


\section{RESULTADOS}

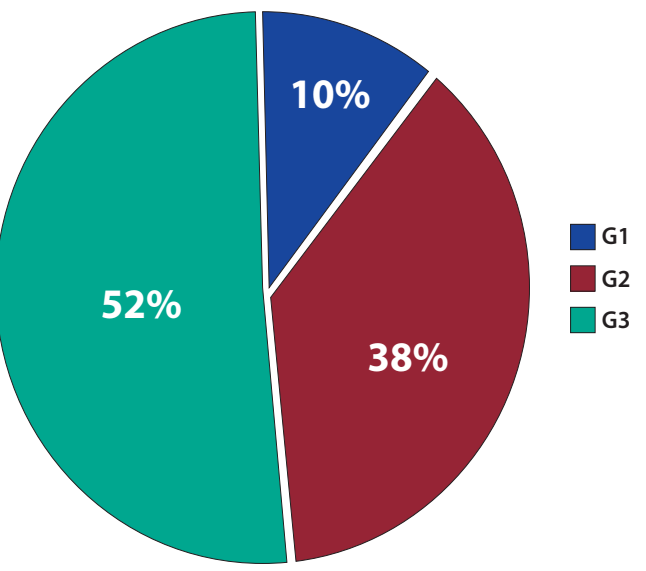

Fuente: INICIB - FAMURP / Hospital Santa Rosa.

Gráfico 1. División por grupo etario.

En el gráfico $N^{\circ} 1$, de una muestra significativa de 180 pacientes, la edad media fue 62 años, se dividió en G1: (1834 años) G2: (35-64 años) y G3: (65 a más) que representan el $10 \%, 38 \%$ y $52 \%$ de la muestra respectivamente.

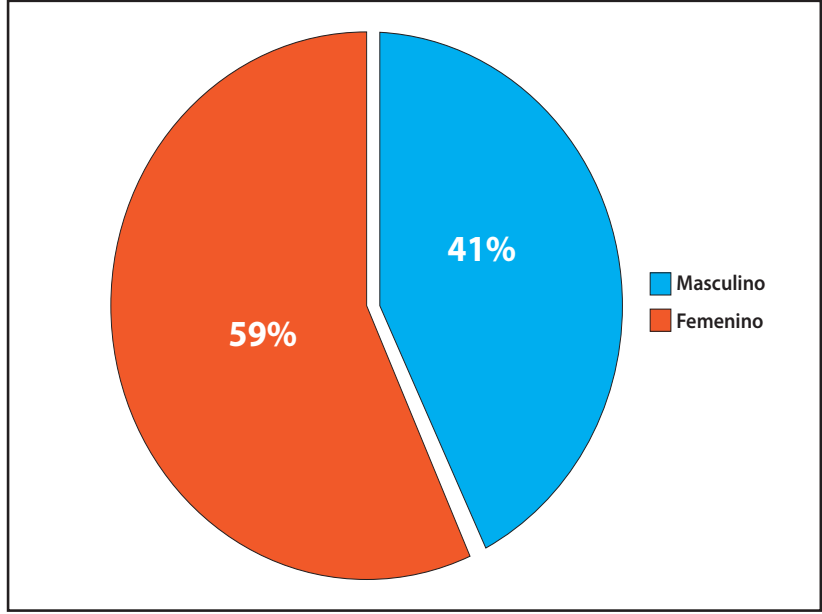

Gráfico 2. División por sexo.

En el gráfico $\mathrm{N}^{\circ} 2$, encontramos una preponderancia parcial del género femenino que representa el $60 \%$ de la muestra, frente a un $40 \%$ del género masculino.

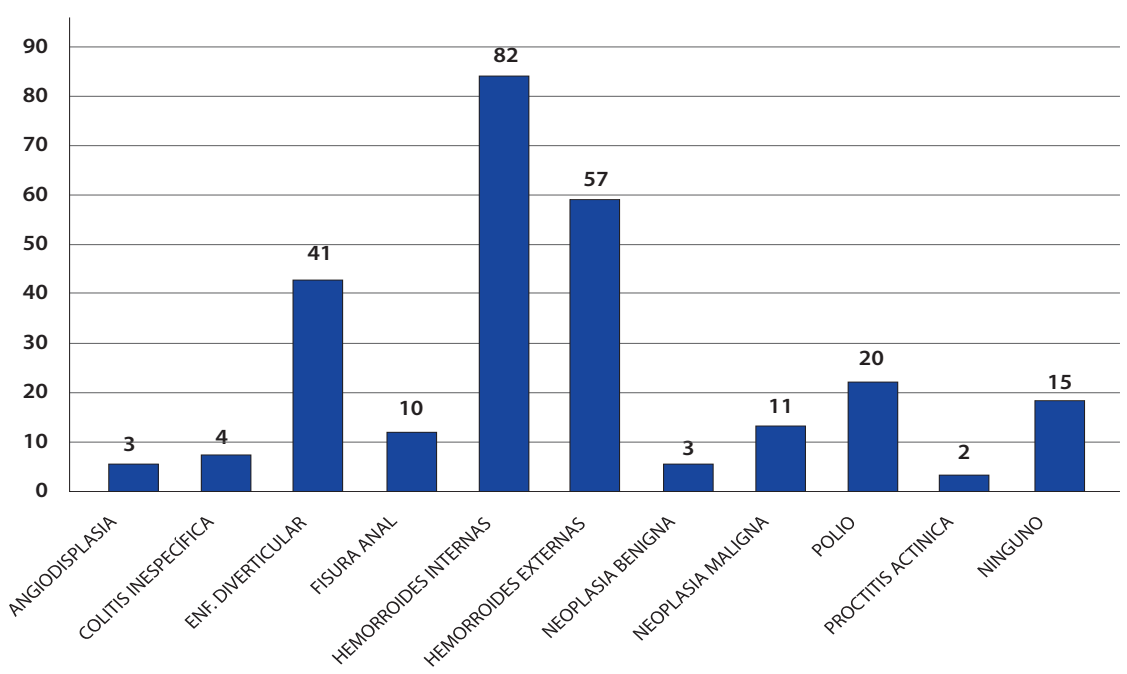

Fuente: INICIB - FAMURP / Hospital Santa Rosa.

Gráfico 3. Tipo de lesión endoscópica en HDB.

Observamos en el gráfico $N^{\circ} 3$, que la lesión más prevalente fue hemorroides internas, diagnosticado en 82 pacientes, lo cual representa (45.55\%) de pacientes afectados por esta enfermedad; seguido de hemorroides externas en 57 pacientes (31.66\%); y la menos prevalente fue proctitis actínica en 2 casos (1.11\%) y no se encontró ninguna lesión en 15 casos que representan $8.33 \%$ de los pacientes.

Tabla 1. Pacientes con diagnóstico de hemorragia digestiva baja, con lesión y sin lesión encontrada por endoscopia baja, hospital Santa Rosa, 2015.

\begin{tabular}{|c|c|c|c|}
\hline & CON LESIÓN & SIN LESIÓN & TOTAL \\
\hline 35 años a más & 162 (94.18\%) & 7 (46.66\%) & 169 (90.37\%) \\
\hline$<35$ años & 10 (5.81\%) & 8 (53.33\%) & 18 (9.62\%) \\
\hline Total & 172 (100\%) & 15 (100\%) & 187 (100\%) \\
\hline Chi cuadrado: 35,813 & $P=, 000$ & Odds ratio: 18,514 & IC: 5,584 \\
\hline
\end{tabular}




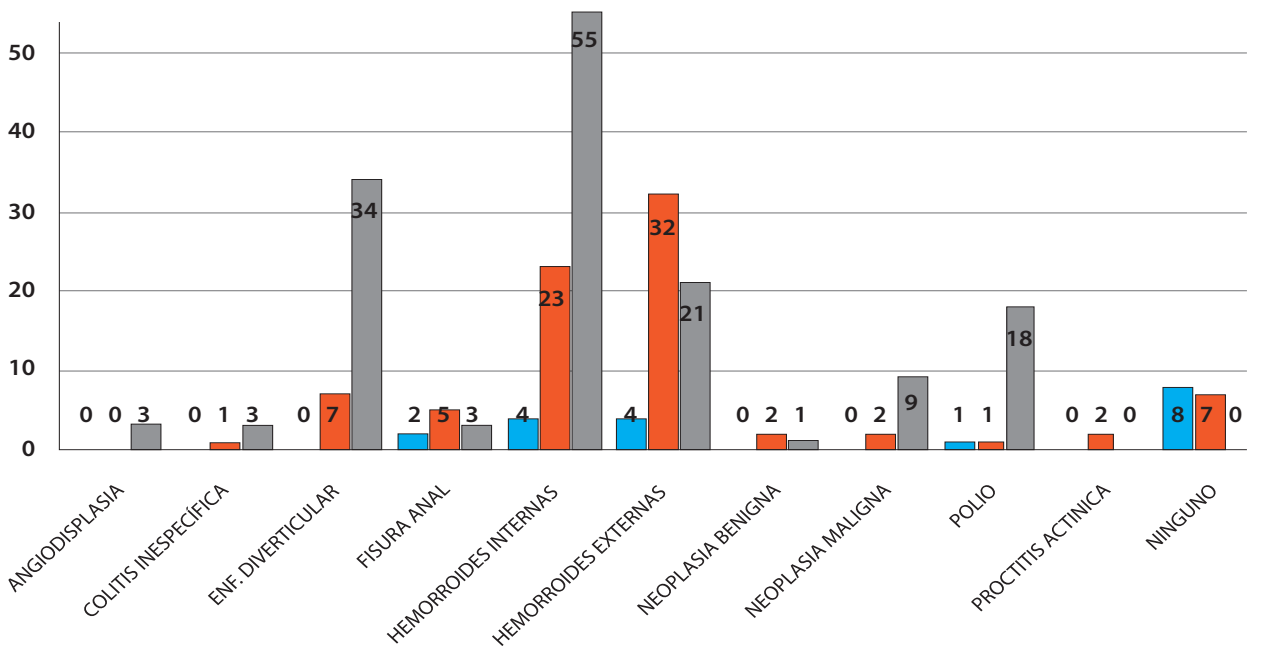

Fuente: INICIB - FAMURP / Hospital Santa Rosa.

Gráfico 4. Tipo de lesión por grupo etario.

En el gráfico $\mathrm{N}^{\circ} 4$, en el grupo etario $\mathrm{G} 3$ predomina hemorroides internas (55 casos), enfermedad diverticular (34 casos), hemorroides externas (21 casos), G2 predomina hemorroides externas (32 casos) y G1 predomina examen si evidencia de lesión endoscópica.

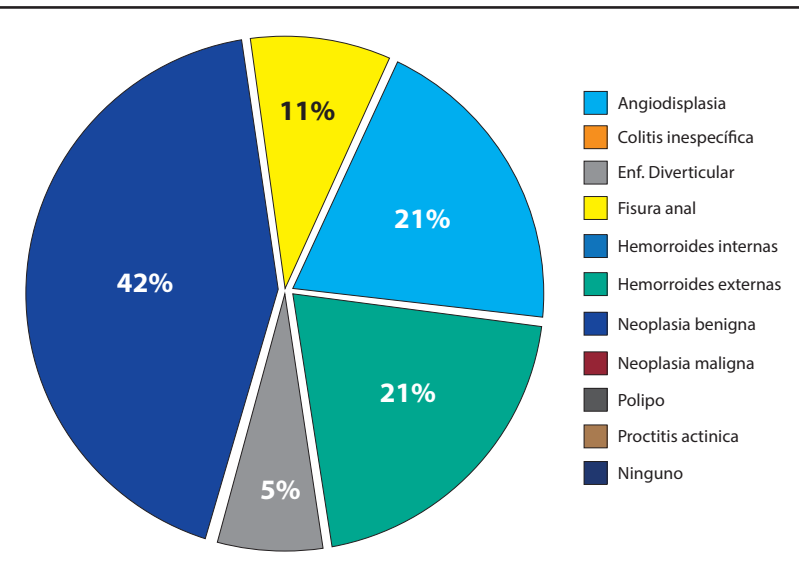

Fuente: INICIB - FAMURP / Hospital Santa Rosa.

Gráfico 5. Tipo de lesión en grupo etario I (18-34 años).

En este gráfico $\mathrm{N}^{\circ} 5$, se observa que en $42 \%$ de los pacientes no se evidenció lesión, en $21 \%$ se encontró hemorroides internas y externas, en menores porcentajes, fisura anal y pólipos.

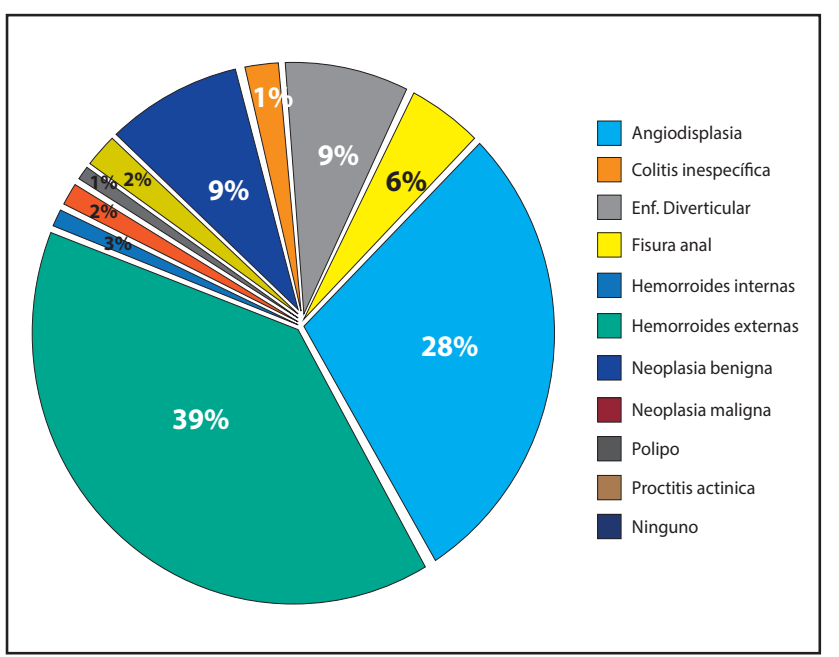

Gráfico 6. Tipo de lesión en grupo etario II (35-64 años).
En el gráfico $\mathrm{N}^{\circ} 6$, obtuvimos un $39 \%$ de pacientes con hemorroides externas, seguido de un $28 \%$ de pacientes con hemorroides internas, en menor porcentaje se obtuvo neoplasia benigna y maligna como $9 \%$ cada uno, fisura anal con $6 \%$; mientras que las demás enfermedades en rangos inferiores al $3 \%$.

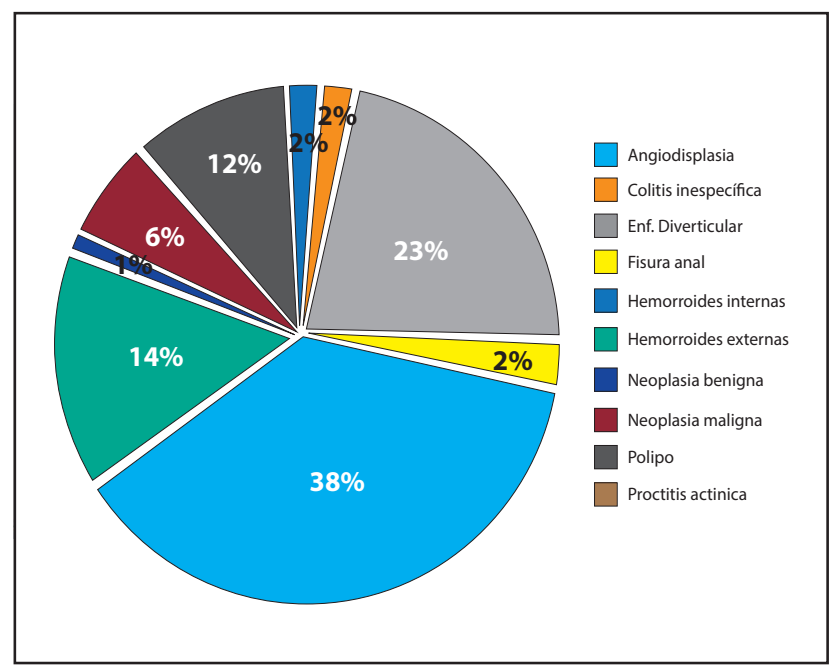

Gráfico 7. Tipo de lesión en grupo etario III (65 años a más).

En el grupo etario III (gráfico $N^{\circ} 7$ ), obtuvimos $38 \%$ de pacientes con hemorroides internas, seguido de $23 \%$ de pacientes con enfermedad diverticular, $14 \%$ con hemorroides externas y $12 \%$ con pólipos, en menor porcentaje se obtuvo a la neoplasia maligna con 6\%; mientras que las demás enfermedades en rangos inferiores al $2 \%$. 


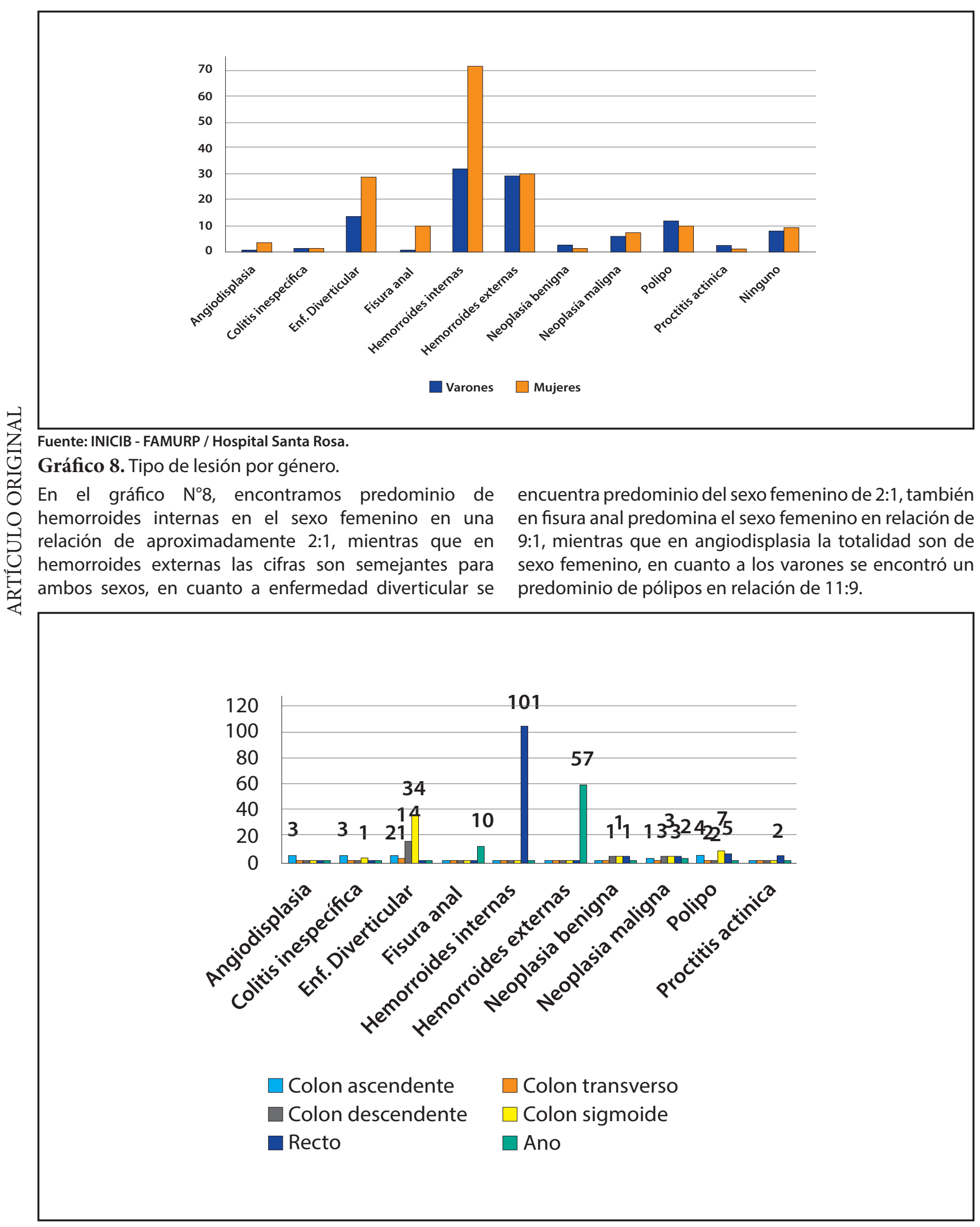

Fuente: INICIB - FAMURP / Hospital Santa Rosa.

Gráfico 9. Localización anatómica más frecuente del tipo de lesión.

Por último, en el gráfico $\mathrm{N}^{\circ} 9$ se obtuvo un predominio de hemorroides internas en el recto con un $100 \%$ de los casos, hemorroides externas en el ano con un $100 \%$ de los casos, enfermedad diverticular: predominando en colon sigmoides (82.92\%), fisura anal (100\%) en el ano, pólipos: predominando en colon sigmoide (35\%), angiodisplasia en colon ascendente con un $100 \%$ de los casos, colitis inespecífica: colon ascendente (75\%), neoplasia maligna: predominando en colon descendente, colon sigmoides y recto (cada uno con $25 \%$ ), proctitis actínica en el recto con un $100 \%$ de casos. 


\section{DISCUSIÓN}

La hemorragia digestiva baja constituye un serio problema de salud para la sociedad actual, ya que tiene una alta tasa de morbilidad, por lo que debemos conocer tanto las causas como el tratamiento más adecuado para la misma. En el hospital Santa Rosa, se obtuvo 342 informes endoscópicos de pacientes con clínica de hemorragia digestiva baja. En la población el promedio etario fue de 62 años, donde el grupo etario más predominante fue el G3 (65 a más), representando más de la mitad de la población estudiada (52\%); dichos resultados concuerdan con la mayoría de los estudios citados en los antecedentes del presente trabajo.

Posteriormente se realizó la valoración de la edad, como condición asociada a la presencia o no de lesión endoscópica, observando la distribución de los pacientes en función a la tabla tetracorica, haciendo posteriormente el cálculo de los parámetros estadísticos correspondientes empezando por el odds ratio cuyo valor resulto de 18,5 y que expresa que aquellos pacientes que tienen 35 a más años, está asociado a la presencia de lesión endoscópica, correspondiente a 18,5 veces más que aquellos pacientes menores de 35 años.

Es por ello que el análisis estadístico a través del test de chi cuadrado cumple la función de determinar la significancia estadística de la distribución obtenida, en este caso en particular resulta siendo positiva la significancia encontrada, lo que implica que la posibilidad de la influencia del azar en la asociación descrita entre ambas variables es inferior al 5\%, lo que resulta ser lo mínimamente esperado. Los resultados tienen una adecuada fuerza de asociación debido a que su valor es mayor de 2 , por lo que en la conclusión resulta determinante la asociación entre ambas variables.

En cuanto al sexo se registra una preponderancia parcial del sexo femenino que representa un 59\% (106 pacientes), frente a un $41 \%$ del sexo masculino (74 pacientes), este resultado nos refleja similitud en comparación a dos estudios, el primero realizado por Geraud Elisa, Ríos Isabel y Francis Pedro en el hospital general del Oeste en Caracas, donde se estudió un total de 389 pacientes, de los cuales el $55.7 \%$ fueron del sexo femenino y $44.3 \%$ del sexo masculino ${ }^{13}$ y el segundo realizado en el por Javier Jesús Suárez Rivera del hospital Clínico Quirúrgico "Julio Trigo" de La Habana en el que se menciona la diferencia entre hombres y mujeres, que en este estudio fue a predominio femenino (8 contra 7$)^{14}$, contrario a lo que la mayoría de la bibliografía indica.

Además, en nuestro estudio encontramos el predominio de hemorroides internas en el sexo femenino en una relación de aproximadamente 2:1, mientras que en hemorroides externas las cifras son semejantes para ambos sexos, en cuanto a enfermedad diverticular se encuentra predominio del sexo femenino de 2:1, también en fisura anal predomina el sexo femenino en relación de 9:1, mientras que en angiodisplasia la totalidad son de sexo femenino, en cuanto a los varones se encontró un predominio de pólipos en relación de 11:9.

Las lesiones endoscópicas más frecuente que se encontraron en los informes de endoscopia baja durante todo el año 2015, dio como resultado: hemorroides internas con un porcentaje de $45.55 \%$ de los pacientes; seguido por hemorroides externas con un porcentaje de $31.66 \%$ de los pacientes; enfermedad diverticular con $22.77 \%$; pólipos con $11.11 \%$; neoplasia maligna con 6.11\%; datos similares presenta en dos estudios, el primero realizado por Cristian Mendez Cordova, Pedro Barrós,José Cabello, Alejandro Bussalleu en el hospital nacional Cayetano Heredia en Lima y el segundo estudio realizado por Geraud Elisa, Ríos Isabel, Francis Pedro en el Hospital General del Oeste en Caracas ${ }^{13}$, en los cuales también se encontraron que los diagnósticos de hemorroides, divertículos y pólipos, encabezaron la incidencia del tipo de lesiones encontradas.

En cuanto a los grupos etarios, encontramos que en el grupo etario I (18-34 años) predomina la ausencia de lesión endoscópica, seguido por hemorroides, como en el estudio de José Alberto Marín A. en México, donde se encontró resultados similares. En el grupo etario II (35-64 años) se encontró predominio de hemorroides con un $67 \%$ y en menor porcentaje se obtuvo neoplasia benigna y maligna como $9 \%$ cada uno, como en el estudio de José Alberto Marín A. En el grupo etario III (65-más años) se encontró predominió de hemorroides con un $52 \%$, seguido de un $23 \%$ con enfermedad diverticular, un $12 \%$ con pólipos, en menor porcentaje se obtuvo neoplasia maligna con $6 \%$, además los casos de angiodisplasia y colitis inespecífica casi en su totalidad pertenecen a este grupo etario; datos similares al estudio de José Alberto Marín.

En cuanto a localización anatómica de las lesiones encontramos un predominio de hemorroides internas en el recto con un $100 \%$ de los casos, hemorroides externas en el ano con un 100\% de los casos, enfermedad diverticular en: colon sigmoides (82.92\%), colon descendente (34.14\%); fisura anal (100\%) en el ano, pólipos en: colon sigmoide (35\%), recto $(25 \%)$, colon ascendente $(20 \%)$, colon transverso (10\%) y descendente (10\%); angiodisplasia en colon ascendente 
con un $100 \%$ de los casos; colitis inespecífica en: colon ascendente (75\%), colon sigmoide (25\%); neoplasia maligna en: colon ascendente (8.33\%), colon descendente (25\%), colon sigmoides (25\%), recto (25\%), ano (16.66\%); proctitis actínica en el recto con un $100 \%$ de los casos, datos similares a lo que refieren los textos teóricos.

\section{CONCLUSIÓN}

En base a los resultados obtenidos concluimos que existe asociación entre la edad y/o el grupo etario con el tipo de lesión endoscópica encontrada, predominando el grupo etario G III, que corresponde a los adultos mayores de 65 años, en el que se encontró la mayor cantidad de hemorroides internas y externas; además de enfermedad diverticular, pólipos y neoplasia maligna en un menor porcentaje; lo cual va acorde con la teoría correspondiente a edad vs tipo de lesión, se encontró la asociación entre la edad del paciente con la presencia o no de lesión endoscópica, con un OR de 18.5; lo cual nos indica que existe 18.5 veces más riesgo que un paciente a mayor edad presente hemorragia digestiva baja encontrada por endoscopia baja, por lo cual esta asociación fue estadísticamente significativa, además se encontró que el sexo femenino es el que presenta con más frecuencia hemorroides internas, mientras que en hemorroides externas las cifras son semejantes para ambos sexos, en cuanto a enfermedad diverticular se encuentra predominio del sexo femenino de 2:1, también en fisura anal predomina el sexo femenino en relación de 9:1, mientras que en angiodisplasia la totalidad son de sexo femenino; en neoplasias se encontró similitud en ambos sexos, a diferencia que en el caso del sexo masculino en lo único que se encontró predominio fue de pólipos en relación de 11:9, también se encontró un predominio de hemorroides internas y externas en un $100 \%$ de los casos en el recto y ano respectivamente; la enfermedad diverticular predominó en el colon sigmoides con $82.9 \%$; pólipos en colon sigmoides con $35 \%$, angiodisplasia en colon ascendente en un 100\% de los casos, colitis inespecífica en el colon ascendente con 75\%; neoplasia maligna encontrándose en el mismo porcentaje en colon descendente, en colon sigmoides y en el recto con un $25 \%$, proctitis actínica en el recto con un $100 \%$ de los casos en los pacientes del servicio de Gastroenterología del hospital Santa Rosa durante el año 2015.

Contribuciones de autoría: La autora participó en la generación, redacción y aprobación final del artículo original, además en la recolección de datos.

Financiamiento: Autofinanciado.

Conflicto de interés: La autora declara no tener conflictos de interés con la publicación de este artículo.

Recibido: 26 de Setiembre del 2018

Aprobado: 19 de Diciembre del 2018

\section{Correspondencia: María Elena Sandoval Pereda}

Dirección: Av. Republica de Panama 350-Dpto 401, Lima-Perú

Teléfono: +51993726650

Correo: marielena-17-89@hotmail.com

\section{REFERENCIAS BIBLIOGRÁFICAS}

\begin{abstract}
1. Rubio $P$, Ortiz Hurtado $H$, Velasco $M$. Función defecatoria y calidad de vida con la cirugía preservadora de esfínteres en el cáncer de recto. Cirugía española: Organo oficial de la Asociación Española de Cirujanos 2003,Vol. 73, №. 1:52-57.
\end{abstract}

2. Miño G, Soriano A, González A, Hervás A J, Naranjo A. Hemorragia digestiva baja grave. Rev And Patol Digest1997: 20: 128-132.

3. Vázquez Sequeiros E, Albillos Martínez A. Protocolo diagnósticoterapéutico de hemorragia digestiva baja. Medicine: Enfermedades del aparato digestivo. Patología intestinal y del peritoneo Medicine: Enfermedades del aparato digestivo. Patología intestinal y del peritoneo 2004, Serie 9, №. 5: 369-372

4. Zuckerman GR, Prakash C, Askin MP, Lewis BS. AGA Technical review on the evaluation and management of occult and obscure gastrointestinal bleeding. Gastroenterol 2000;118(1):201-221.

5. Church J. Adenoma Detection Rate and the Quality of Colonoscopy: The Sword has Two Edges. Dis Colon Rectum 2008; 51: 520-523.

6. Kovacs TOG, Jensen DM. Endoscopic control of gastroduodenal hemorrhage. Ann Rev Med 1987;38:267- 277.
7. Junquera F, Quiroga S, Saperas E. Et al. Accuracy of helical computed tomographic angiography for the diagnosis of colonic angiodysplasia. Gastroenterology 2000; 119(2):293-299.

8. Chol YB, Lim KS. Therapeutic laparoscopy for abdominal trauma.Surg Endosc. 2003;17(3):421-7

9. J.J. Farrell,L.S. Friedman Review article: the management of lower gastrointestinal bleeding Aliment Pharmacol Ther, 21 (2005), pp. 12811298

10. R.E. Davila,E. Rajan,D.G. Adler,J. Egan,W.K. Hirota,J.A. Leighton ASGE Guideline: the role of endoscopy in the patient with lower-Gl bleeding Gastrointest Endosc, 62 (2005), pp. 656-660

11. F. Rodriguez Moranta,A. Berrozpe,J. Guardiola Diagnostic performance of colonoscopy in lower gastrointestinal bleeding Gastroenterol Hepatol, 34 (2011), pp. 551-557

12. L.L. Strate,C.R. Naumann The role of colonoscopy and radiological procedures in the management of acute lower intestinal bleeding. Clin Gastroenterol Hepatol, 8 (2010), pp. 333-343 
13. Geraud Elisa, Ríos Isabel, Francis Pedro, Ollarves María, Gori María Folkmanas William et al. Hallazgos endoscopicos en pacientes con hemorragia digestiva inferior en el Hospital General del Oeste durante los años 2003-2008. Gen [Internet]. 2010 Sep [citado 2018 Mar 2] ; 64( 3 ): 180-185. Disponible en: http://www.scielo.org.ve/scielo.php?script=sci arttext\&pid=S0016-35032010000300007\&lng=es
14. Suárez J. Caracterización-de-pacientes fallecidos-por-hemorragiadigestiva-baja. Revista portales médicos.com [Internet]. 2014 Feb. [citado 2018 Feb Disponible en: https://www.revista-portalesmedicos.com/ revista-medica/publicaciones/gastroenterologia/caracterizacion-depacientes-fallecidos-por-hemorragia-digestiva-baja/

Consulte la Versión Electrónica de la Revista:

Facultad de Medicina Humana Universidad Ricardo Palma

http://revistas.urp.edu.pe/index.php/RFMH<smiles>C1=C2CC=[C+]2CC1</smiles>

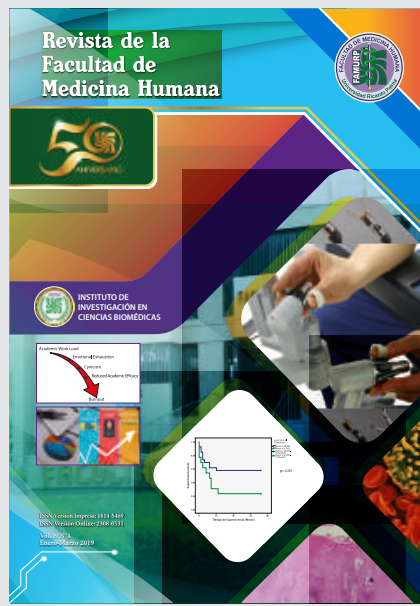

